\title{
Thyroid storm after mitral valve repair in a patient with Becker muscular dystrophy
}

\author{
Kazuhiro Yamazaki ${ }^{1}$, Kenji Minakata ${ }^{2}$, Takeichiro Nakane ${ }^{3}$, Masahide Kawatou ${ }^{1}$, Kenji \\ Minatoya $^{4}$, and Ryuzo Sakata ${ }^{5}$ \\ ${ }^{1}$ Graduate School of Medicine, Kyoto University \\ ${ }^{2}$ Kyoto University Graduate School of Medicine \\ ${ }^{3}$ Kyoto University \\ ${ }^{4}$ Natl Cerebral \\ ${ }^{5}$ Affiliation not available
}

October 22, 2020

\begin{abstract}
A 40-year-old male with Becker muscular dystrophy presented with severe mitral regurgitation and underwent mitral valve repair. Following the surgery, the patient became tachycardic, and developed a continuous high grade-fever and hyperbilirubinemia. The patient's condition worsened and we eventually tested his thyroid levels and discovered abnormally high thyroid levels. After diagnosing a severe thyroid storm, the patient was treated with oral administration of Lugol's iodine and thiamazole, as well as an intravenous steroid, which led to an immediate improvement of symptoms. The incidence of thyroid storm after open-heart surgery is extremely rare but highly life-threatening if unrecognized.
\end{abstract}

\section{Introduction}

Thyroid storm is a rare condition that involves severe clinical manifestations of hyperthyroidism. It is lifethreatening and requires urgent treatment. In particular, thyroid storm after open heart surgery is extremely rare, and there have been very few cases reported in the literature. Herein, we report a case of thyroid storm that developed after mitral valve repair surgery, which was very difficult to manage because we were initially unable to diagnose it.

\section{Case Report}

A 40-year-old man was admitted to our hospital for treatment of recurrent heart failure. Twelve years prior, he had been diagnosed with dilated cardiomyopathy and Becker muscular dystrophy. After the initial diagnosis, the patient's heart failure gradually worsened and the patient was referred to our hospital for further treatment. On presentation, the patient's clinical features were as follows: body temperature of $36.4^{\circ} \mathrm{C}$, blood pressure of $82 / 54 \mathrm{mmHg}$, pulse rate of 80 beats/min, Levine $3 / 6$ systolic heart murmur on auscultation. An electrocardiogram showed normal sinus rhythm and a heart rate of 82 beats/min. Echocardiography showed ventricular dilatation and decreased contractility (ejection fraction; EF, 25\%), with severe functional mitral regurgitation due to leaflet tethering. Regarding limb strength, Gowers' sign was present and he had difficulty crouching.

We performed mitral annuloplasty (28mm SJM Rigid Saddle Ring; Abbott, St. Paul, MN, USA), as well as papillary muscle approximation with posterior left ventriculoplasty. The patient was weaned from cardiopulmonary bypass with high dose of inotropic support as well as intra-aortic balloon pumping (IABP). The cardiopulmonary bypass time was $179 \mathrm{~min}$, and the aortic cross-clamp time was $127 \mathrm{~min}$. 
On admission to the intensive care unit, the heart rate was around 130-140beats/min, blood pressure was $100 / 50 \mathrm{mmHg}$, pulmonary artery pressure was $30 / 20 \mathrm{mmHg}$, and cardiac index (CI) was $3.0 \mathrm{~L} / \mathrm{min} / \mathrm{m}^{2}$. On postoperative day (POD) 1 , CI was above $3.0 \mathrm{~L} / \mathrm{min} / \mathrm{m}^{2}$, and IABP was discontinued. Subsequently, the doses of dopamine and milrinone were gradually decreased and completely discontinued on POD 8. After discontinuing these drugs, the patient's heart rate remained at around 80beats/min under sedation, but when he woke up, he became tachycardic (approximately 120 beats/min). Echocardiography showed decreased contractility (EF, $\sim 30 \%$ ), without mitral regurgitation.

Following the surgery, the patient developed a continuous fever $\left(39.0^{\circ} \mathrm{C}\right.$ or higher: Figure $\left.1 \mathrm{~A}\right)$. We suspected malignant hyperthermia, however, his serum creatine kinase level peaked at approximately $1500 \mathrm{U} / \mathrm{L}$, and there was no muscular rigidity. Moreover, the patient's bilirubin level was elevated after the operation and continued to rise (predominantly direct hyperbilirubinemia). During this time, serum transaminase levels (aspartate transaminase and alanine transaminase) were relatively normal (both approximately 30-60 U/L) and alkaline phosphatase levels were only mildly elevated (approximately $350 \mathrm{U} / \mathrm{L}$ ). Computed tomography and abdominal ultrasonography showed no abnormalities in the hepatobiliary system. It appeared unlikely that the patient had developed hyperbilirubinemia due to liver shock liver given his preserved cardiac output throughout the postoperative course. Of note, the patient continued to have normal kidney function. Therefore, plasmapheresis was performed on POD 10 when total bilirubin reached $20 \mathrm{mg} / \mathrm{dL}$. Despite this, his total bilirubin level remained high at approximately $10 \mathrm{mg} / \mathrm{dL}$ (Figure 1B). The patient also developed watery diarrhea, but he was negative for Clostridium difficile .

The patient continued to be febrile, produced copious sputum due to pneumonia, and showed signs of congestive heart failure, and we decided to sedate him and put him on ventilator support. When we performed a tracheotomy to do so, we noticed well-developed blood vessels around the thyroid gland. In fact, these vessels resulted in significant bleeding that was difficult to manage.

After the tracheotomy, the patient continued to have delirium, and his mental status did not improve even after sedation was discontinued. At this time, various hormone tests were performed, and abnormal levels of thyroid hormone were observed (thyroid-stimulating hormone, $<0.005 \mu \mathrm{IU} / \mathrm{mL}$; free $\mathrm{T}_{4}>7.770$ $\mathrm{ng} / \mathrm{dL}$; free $\mathrm{T}_{3} 9.98 \mathrm{pg} / \mathrm{mL}$ ). Based on these figures, the patient was diagnosed with thyroid storm. Lugol's iodine and thiamazole (30 mg/day) were administered orally. In addition, a dose of steroid (hydrocortisone sodium succinate, $100 \mathrm{mg}$ ) was administered intravenously every 8 hours. As a result, the patient's mental status immediately improved, his heart rate decreased from 120-140 beats/min to 100beats/min, and his fever subsided (Figure 1). Technetium scintigraphy of the thyroid was performed on POD 37, and showed abnormally low uptake (0.01\%: Figure 2), confirming destructive thyrotoxicosis. On POD 42, free $\mathrm{T}_{4}(4.080$ $\mathrm{ng} / \mathrm{dL})$ and free $\mathrm{T}_{3}(2.48 \mathrm{pg} / \mathrm{mL})$ were improved and we discontinued Lugol's iodine, and reduced the dose of thiamazole to $15 \mathrm{mg} /$ day. The tracheostomy was closed, and the patient was transferred to a long-term care facility to continue rehabilitation.

\section{Discussion}

Thyroid storm may be caused by various stresses, such as infection, trauma, and surgery in a patient with untreated or poorly controlled underlying thyroid disease, resulting in an overproduction of thyroid hormones that can lead to multiple organ malfunctions. In Japan, the annual incidence of thyroid storm is 0.2 per 100,000 , and the mortality rate of patients from thyroid storm is approximately $11 \%$ [1]. Thus, thyroid storm is a rare but life-threatening condition requiring urgent treatment.

In general, thyroid storm is diagnosed according to the Burch-Wartofsky criteria[2]. Retrospectively, it was obvious that our patient had all the telltale signs, and we calculated the patient's score to be 105(a score greater than 60 is consistent with thyroid storm). Therefore, it would have been easy to make a diagnosis of thyroid storm had we been more aware of the condition. Unfortunately, it was not until we performed a tracheotomy that we were led to suspect hyperthyroidism due to the abnormal development of blood vessels around the thyroid. Since we did not have previous knowledge or experience of a thyroid storm, we were trying to deal with each symptom individually until the correct diagnosis. 
The differential diagnosis of thyroid storm includes cardiogenic shock, malignant hyperthermia, and sepsis. In this case, we had performed mitral repair on the patient due to reduced cardiac function. Therefore, we initially assumed that the respiratory failure and tachycardia were caused by severe heart failure. In addition, our patient had Becker muscular dystrophy as a co-morbidity, which has known to be a risk factor for malignant hyperthermia related to general anesthesia[3]. This condition also made the diagnosis difficult.

There have only been a few case reports of thyrotoxicosis and thyroid storm in the peri-operative period of open-heart surgery, all of which occurred in coronary artery bypass grafting and were associated with coronary artery spasm and increased myocardial oxygen demand[4-8]. To the best of our knowledge, this is the first case report of thyroid storm occurring after cardiac valve surgery.

Patient consent statement. This patient signed informed consent to participate in our study.

Availability of data. All the co-authors have full access to the patient data. Also, the datasets during and/or analyzed during the current study available from the corresponding author on reasonable request.

Conflicts of interest. The authors declare that they have no competing interests.

Funding. None

Authors' contributions . All authors contributed to the writing of the final manuscript.

\section{References}

1. Akazumi T. Thyroid storm: A Japanese perspective. Thyroid . 2018; 28:32-40.

2. Burch HB, Wartofsky L. Life-threatening thyrotoxicosis, thyroid storm.Endocrinol Metab Clin N Am. 1993;23:263-277.

3. Gurnaney H, Brown A, Litman RS. Malignant hyperthermia and muscle dystrophies. Aneth Analg 2009;109:1043-1048.

4. Redahan C, Karski JM. Thyrotoxicosis factitia in a post-aortocoronary bypass patient. Can J Anaesth 1994; 41:969-72.

5. Lee SM, Jung ST, Hahm JR, Im SI, Kim SK, Lee KJ. et al. Thyrotoxicosis with coronary spasm that required coronary artery bypass surgery.Intern Med. 2007;46:1915-1918.

6. Bish LT, Bavaria JE, Augoustides J. Thyroid storm after coronary artery bypass grafting. J Thorac Cardiovasc Surg 2010;140:e67-9.

7. Kim HJ, Jung TS, Hahm JR, Hwang SJ, Lee SM, Jung JH. et al. Thyrotoxicosis-induced acute myocardial infarction due to painless thyroiditis. Thyroid. 2011;10:1149-51.

8. Lee JH. Thyroid storm after coronary artery bypass surgery: a case report. J Cardiothorac Surg. 2020;15:22. doi: 10.1186/s13019-020-1044-2.

\section{Figure Legends}

Figure 1. A) Total bilirubin and Direct bilirubin: Bilirubin level was high from the day after surgery. B) Body temperature: $39.0{ }^{\circ} \mathrm{C}$ or higher continuous fever following surgery. After treating the thyroid storm, all symptoms immediately improved.

Figure 2. Technetium scintigraphy of the thyroid showed abnormally low uptake $(0.01 \%)$ and confirmed destructive thyrotoxicosis. 

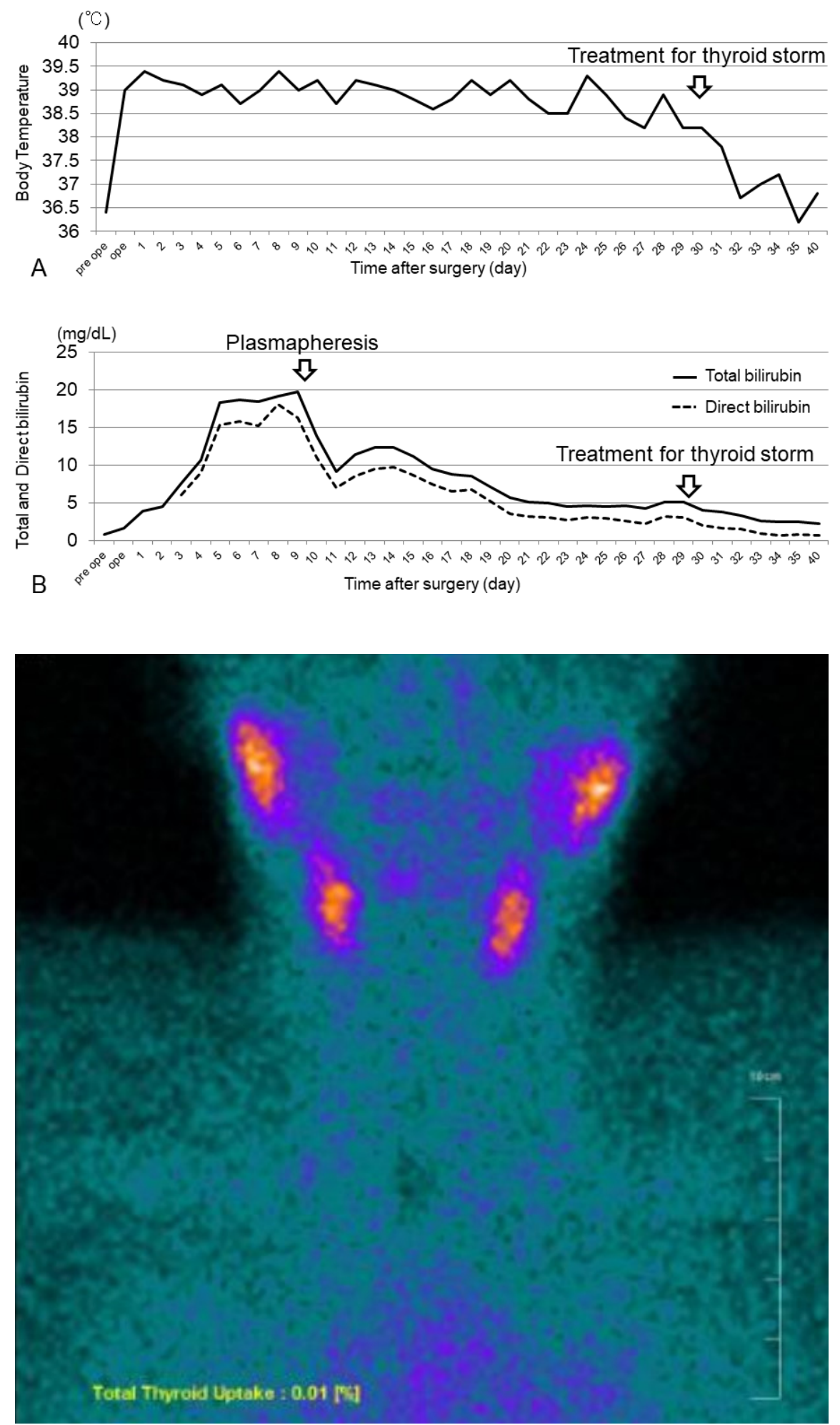